\title{
Patrones de distribución de la flora vascular acuática estricta en el estado de Tamaulipas, México
}

\section{Distributional patterns of the strictly aquatic vascular flora in the state of Tamaulipas, Mexico}

\author{
Arturo Mora-Olivo ${ }^{1 *}$, José Luis Villaseñor ${ }^{2}$, Isolda Luna-Vega ${ }^{3}$ y Juan J. Morrone ${ }^{4}$ \\ ${ }^{1}$ Instituto de Ecología y Alimentos, Universidad Autónoma de Tamaulipas, 13 Blvd. Adolfo López Mateos 928, Ciudad Victoria 87040, Tamaulipas, \\ México. \\ ${ }^{2}$ Departamento de Botánica, Instituto de Biología, Universidad Nacional Autónoma de México. Apartado postal 70-367, 04510 México, D. F., \\ México. \\ ${ }^{3}$ Departamento de Biología Evolutiva, Facultad de Ciencias, Universidad Nacional Autónoma de México , Apartado postal 70-399, 04510 México, \\ D.F., México. \\ ${ }^{4}$ Museo de Zoología "Alfonso L. Herrera”, Departamento de Biología Evolutiva, Facultad de Ciencias, Universidad Nacional Autónoma de México, \\ Apartado postal 70-399, 04510 México, D. F., México. \\ *Correspondencia: amorao@uat.edu.mx
}

\begin{abstract}
Resumen. Se analizaron los patrones de riqueza y distribución de las plantas vasculares acuáticas estrictas en el estado de Tamaulipas, México. Se registraron 93 especies, 62 de las cuales son típicas de ambientes lénticos. La subcuenca Río Tamesí fue la que registró el mayor número de especies $(68,73.1 \%)$. No se registraron especies endémicas del estado, aunque existen 2 especies restringidas al territorio mexicano (Lobelia purpusii y Oserya coulteriana). Se consideran como raras 29 especies $(31.2 \%)$ por presentarse en una sola subcuenca y sólo 2 especies están distribuidas ampliamente en la mayoría de éstas (Bacopa monnieri y Echinodorus berteroi). Un análisis de parsimonia de endemismos (PAE) de las subcuencas hidrológicas del estado con base en la presencia compartida de especies reveló que las plantas acuáticas estrictas presentan un patrón de distribución anidado. Se concluye que las plantas vasculares acuáticas estrictas de Tamaulipas son parte de una misma unidad florística y que su distribución obedece a gradientes altitudinales, latitudinales y climáticos.
\end{abstract}

Palabras clave: hidrófitas estrictas, análisis de parsimonia de endemismo, plantas tamaulipecas.

\begin{abstract}
Patterns of distribution and richness of strictly aquatic vascular plants of the state of Tamaulipas, Mexico, were analyzed. We registered 93 species of strictly aquatic vascular plants, which 62 are typical from lentic environments. The Río Tamesí sub-basin contains the highest number of species $(68,73.1 \%)$. There are no endemic species to the state of Tamaulipas, but we found 2 species endemic to Mexico (Lobelia purpusii and Oserya coulteriana). Twenty nine species (31.2\%) are considered rare, because they are present in a single sub-basin; only 2 species are widely distributed in most of them (Bacopa monnieri and Echinodorus berteroi). A parsimony analysis of endemicity (PAE) of the hydrological subbasins represented in this state based on the shared presence of species suggested that the strictly aquatic vascular plants have a nested distributional pattern. We conclude that these plants in Tamaulipas are part of the same floristic unit and that their distribution follow altitudinal, latitudinal and climatic gradients.
\end{abstract}

Key words: strictly hydrophytes, parsimony analysis of endemicity, Tamaulipan plants.

\section{Introducción}

La búsqueda de regularidades en la distribución de los seres vivos es uno de los objetivos principales de la biogeografía (Zunino, 2005). Los sistemas acuáticos en general son poco diversos, aun en ambientes tropicales (Rzedowski, 1991a; Crow, 1993), y las especies de plantas acuáticas usualmente poseen áreas de distribución amplias, muchas de ellas cosmopolitas

Recibido: 30 mayo 2007; aceptado: 12 febrero 2008 o subcosmopolitas (Santamaría, 2002), debido en parte a su amplia tolerancia ecológica. En contraste con las plantas terrestres, las hidrófitas se caracterizan por tener una escasa diversificación taxonómica y genética a niveles supraespecíficos (Sculthorpe, 1985; Santamaría, 2002). Comparado con otros grupos vegetales, el endemismo en las plantas acuáticas es bajo, como también sucede con bejucos, algunos helechos y orquídeas (Rzedowski, 1991b; McGlone et al., 2001). La dispersión a larga distancia por aves ha sido la causa que más frecuentemente se ha utilizado para explicar las distribución discontinua de especies 
acuáticas (Santamaría y Klaassen, 2002; Les et al., 2003), aunque ésta no siempre ha explicado satisfactoriamente dichas distribuciones en el caso de grandes barreras geográficas, como las disyunciones intercontinentales, que podrían deberse a eventos históricos como la vicarianza y la deriva continental (Santamaría, 2002).

Los métodos empleados para elaborar trabajos biogeográficos para México han variado desde los netamente descriptivos hasta los cuantitativos (Murguía y Rojas, 2001). Los análisis fenéticos (Balleza et al., 2005), panbiogeográficos (Contreras-Medina y Eliosa, 2001; Morrone et al., 2002) y cladísticos (ContrerasMedina et al., 2007a; Escalante et al., 2007) han sido una herramienta útil para definir regiones, provincias y distritos biogeográficos. El método conocido como análisis de parsimonia de endemismos o PAE (por sus siglas en inglés) clasifica las áreas estudiadas (análogas a taxones en análisis filogenéticos) con base en los taxones compartidos (análogos a caracteres) generando un cladograma que muestra jerárquicamente las áreas con biotas similares (Rosen, 1988; Morrone y Crisci, 1995). En México, el PAE se ha usado con diferentes unidades geográficas y grupos taxonómicos; por ejemplo, áreas fragmentadas y plantas del bosque mesófilo de montaña (Luna et al., 1999, 2000), provincias florísticas y cactáceas columnares (DávilaAranda et al., 2002), cuadrantes y plantas vasculares endémicas de la península de Yucatán (Espadas et al., 2003), cuadrantes y magnoliofitas endémicas de la Reserva de la Biosfera Tehuacán-Cuicatlán (Méndez-Larios et al., 2005), sistemas hidrológicos y helmintos parásitos de peces (Aguilar-Aguilar et al., 2003, 2005), transectos y aves del oeste del país (García-Trejo y Navarro-Sigüenza, 2004), cortes latitudinales y mamíferos de la Sierra Madre Oriental (León-Paniagua et al., 2004), provincias biogeográficas e insectos (Corona et al., 2005), provincias bióticas y mariposas (Luis et al., 2005), estados y árboles tropicales de México (Cué-Bär et al., 2006) o estados, provincias biogeográficas, cuadrantes y gimnospermas de México (Contreras-Medina et al., 2007b). Los estudios biogeográficos relacionados con plantas acuáticas son escasos (Stuckey, 1993; Santamaría, 2002; Les et al., 2003; Sawada et al., 2003), especialmente en México (Lot y Novelo, 1992), donde el único trabajo que aplica el PAE es el de Huidobro et al. (2006).

En Tamaulipas convergen porciones de reinos, regiones y provincias propuestos por diferentes autores. Por ejemplo, desde el punto de vista florístico, Rzedowski (1978) señala que en el estado confluyen los reinos holártico y neotropical; están representadas 3 regiones (Mesoamericana de Montaña, Xerofítica Mexicana y Caribea) y 4 provincias (Sierra Madre Oriental, Altiplanicie, Planicie Costera del Noreste y Costa del Golfo de México).
Morrone et al. (2002), con plantas y animales, también ubican en su territorio las regiones neártica y neotropical y 4 provincias biogeográficas (Sierra Madre Oriental, Altiplano Mexicano, Tamaulipas y Golfo de México). Por su parte, la Comisión Nacional para el Conocimiento y Uso de la Biodiversidad (CONABIO) (Arriaga et al., 1997) propuso un sistema combinado basado en plantas (Rzedowski, 1978), animales (Casas-Andreu y ReynaTrujillo, 1990; Ramírez-Pulido y Castro-Campillo, 1990) y rasgos morfotectónicos (Ferrusquía-Villafranca, 1990), encontrando para el país 19 provincias biogeográficas. En Tamaulipas se localizan porciones de las provincias Sierra Madre Oriental, Altiplano Sur, Tamaulipeca y Golfo de México (Fig. 1).

Tamaulipas cuenta actualmente con un inventario actualizado de su flora vascular acuática (Mora-Olivo y Villaseñor, 2007). Con base en esta información, el

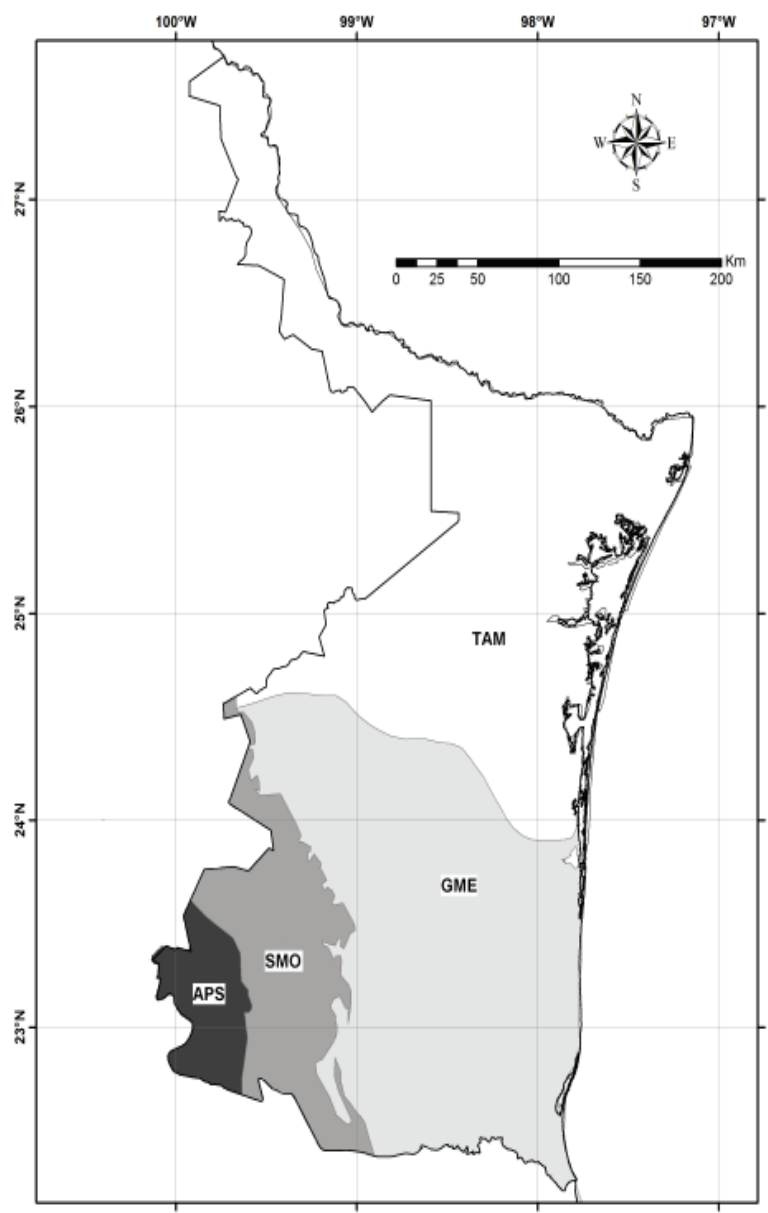

Figura. 1. Provincias biogeográficas (CONABIO, 2007) que se localizan en el estado de Tamaulipas. TAM = Tamaulipeca, GME $=$ Golfo de México, SMO = Sierra Madre Oriental, APS $=$ Altiplano Sur. 
objetivo del trabajo es evaluar el patrón de distribución de las plantas vasculares acuáticas estrictas usando un análisis de parsimonia de endemismos, con el fin de detectar áreas particularmente ricas en especies exclusivas que las diferencian como zonas particularmente interesantes por la distribución restringida de tales especies.

\section{Material y métodos}

Área de estudio. Tamaulipas tiene una superficie de 78,380 $\mathrm{km}^{2}$ y se localiza entre los $22^{\circ} 12^{\prime} 31^{\prime \prime}$ y $27^{\circ} 40^{\prime} 42^{\prime \prime}$ de N y $\operatorname{los} 97^{\circ} 08^{\prime} 38^{\prime \prime}$ y $100^{\circ} 08^{\prime} 52^{\prime \prime}$ O. Colinda al norte con el estado de Texas de los Estados Unidos de América, al sur con los estados de Veracruz y San Luis Potosí, al este con el golfo de México y al oeste con el estado de Nuevo León (Fig. 2). La mayor parte del territorio tamaulipeco se eleva poco sobre el nivel del mar, con excepción de la Sierra Madre Oriental, que en su parte más alta llega a los 3000 m. Por su localización geográfica y su orografía, Tamaulipas presenta una gran diversidad de climas, que van desde los subhúmedos y húmedos, con lluvias en verano en la zona sur-sureste, hasta climas templados en el altiplano tamaulipeco y serranías, que varían de húmedos a secos según la altitud (INEGI, 2001).

En el estado de Tamaulipas se encuentran representadas total o parcialmente 13 cuencas hidrológicas y 50 subcuencas (SPP, 1982; CNA, 1998; INEGI, 2001) (Cuadro 1). Los principales cuerpos de agua son los ríos Bravo, San Fernando, Soto la Marina y Tamesí, y las lagunas Madre y San Andrés (SPP, 1982) (Fig. 2). Se ha registrado la presencia de manglares, tulares y carrizales en el estado (Miranda y Hernández-X., 1963), y además la característica vegetación de ríos, presas, canales, lagunas y charcos (Martínez y Novelo, 1993). Un inventario reciente reporta 426 especies de plantas vasculares asociadas con humedales, de las cuales 93 se consideran acuáticas estrictas nativas (Mora-Olivo y Villaseñor, 2007).

Con base en recolecciones de campo, revisión de ejemplares de herbario (UAT, MEXU, ENCB, XAL, TEX), acceso a bases de datos (REMIB, W3TROPICOS; Novelo, inédito; Villaseñor, inédito) y consulta de bibliografía especializada (e.g. Lot et al., 1986, 1999; González, 1989; Tucker, 1994; Olvera, 1996; Novelo y Philbrick, 1997; Pérez-García et al., 1999; Haynes y Holm-Nielsen, 1994, 2003), se elaboró una base de datos con 1376 registros georreferidos de especies de plantas vasculares acuáticas de Tamaulipas. Con esta información se determinó la presencia de 93 especies de plantas acuáticas estrictas no introducidas (Mora-Olivo y Villaseñor, 2007), en las cuencas y subcuencas en que se divide oficialmente el estado desde el punto de vista hidrológico (SPP, 1982;
CNA, 1998; INEGI, 2001). Algunas cuencas y subcuencas no registraron datos y otras tuvieron un número reducido de especies; en estos casos, se incluyeron en sus contrapartes vecinas que registraron un mayor número de especies como recomiendan Glasby y Álvarez (1999). Un caso especial fue el de la subcuenca Tula 1, en la cual sólo se registraron 3 especies, pero se tomó en cuenta de manera independiente dadas sus características ambientales, pues forma parte del Altiplano Mexicano. De esta manera, el número final de cuencas y subcuencas analizadas se redujo a 7 y 15 respectivamente (Fig. 2). Se decidió usar subcuencas en lugar de cuencas, debido a que algunas de éstas son muy extensas y en muchos casos ocupan distintas latitudes, longitudes y provincias bióticas. Otros estudios biogeográficos en México que utilizaron

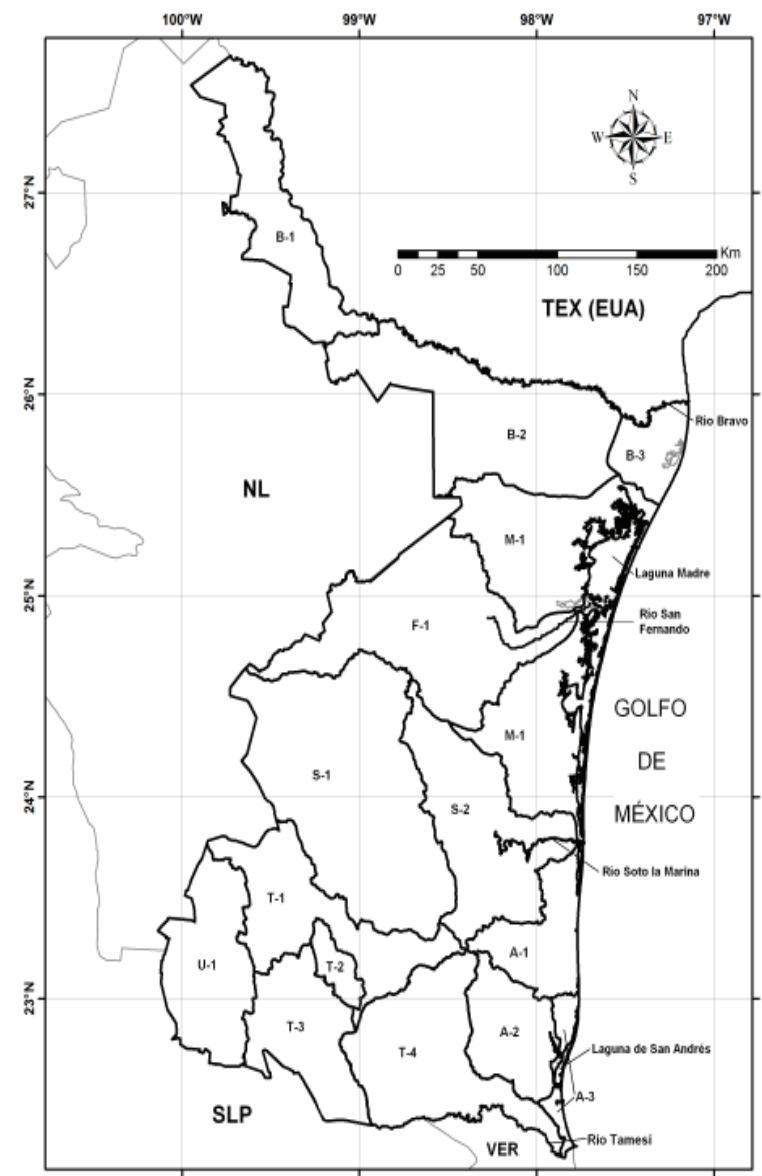

Figura 2. Localización de las subcuencas hidrológicas y de los principales ríos en Tamaulipas. TEX $=$ Texas, EUA = Estados Unidos de América, NL = Nuevo León, SLP $=$ San Luis Potosí, VER $=$ Veracruz. Subcuencas: B- $1=$ Bravo 1 , B-2 $=$ Bravo 2, B$3=$ Bravo 3, M-1 = Madre 1, F-1 = Fernando $1, \mathrm{~S}-1=$ Soto $1, \mathrm{~S}-2$ = Soto 2, A-1 = Andrés 1, A-2 = Andrés 2, T-1 = Tamesí 1, T-2 = Tamesí 2, Tamesí-3 = Tamesí 3, T-4 = Tamesí 4. U-1 = Tula 1 . 
Cuadro 1. Número de registros, especies de plantas vasculares acuáticas estrictas y especies endémicas de México en las cuencas (7) y subcuencas hidrológicas (15) en que se dividió el estado de Tamaulipas

\begin{tabular}{lllll}
\hline Cuenca (especies) & Subcuenca & $\begin{array}{l}\text { Superficie } \\
\left(\mathrm{km}^{2}\right)\end{array}$ & Registros & $\begin{array}{l}\text { Especies } \\
\text { (endémicas) }\end{array}$ \\
\hline Río Bravo (23) & Bravo 1 & 4895.3 & 8 & 8 \\
& Bravo 2 & 8012.1 & 34 & 16 \\
& Bravo 3 & 1656.7 & 45 & 27 \\
Laguna Madre (27) & Madre 1 & 9699.5 & 71 & 10 \\
Río San Fernando (10) & Fernando 1 & 8814.3 & 9 & $26(1)$ \\
Río Soto la Marina (39) & Soto 1 & 12146.7 & 97 & 32 \\
Laguna San Andrés (46) & Soto 2 & 6345.8 & 80 & 22 \\
& Andrés 1 & 2709.6 & 51 & 18 \\
Río Tamesí (75) & Andrés 2 & 2957.0 & 30 & 28 \\
& Andrés 3 & 686.7 & 32 & $28(1)$ \\
& Tamesí 1 & 5060.8 & 198 & $18(1)$ \\
\hline & Tamesí 2 & 752.8 & 54 & 27 \\
& Tamesí 3 & 3974.6 & 36 & 68 \\
\hline
\end{tabular}

unidades hidrológicas son los de Aguilar-Aguilar et al. (2005), Espinosa y Huidobro (2005) y Espinosa et al. (2006), que trabajaron con helmintos acuáticos, peces y plantas terrestres, respectivamente. Para correlacionar los datos de riqueza de especies con el número de registros y la superficie en cada una de las subcuencas analizadas, se realizaron análisis de regresión simple utilizando el programa JMP versión 5.0.1a (SAS, 2002).

Se utilizó la metodología del PAE para clasificar a las subcuencas con base en la presencia compartida de 2 o más especies de plantas vasculares acuáticas estrictas (Crisci et al., 2000; Escalante y Morrone, 2003). Con los datos de presencia-ausencia de estas especies en cada una de las subcuencas, se creó una matriz a la que se le incluyó un área hipotética con "0" para enraizar los cladogramas (Morrone, 1994) (Cuadro 2). La matriz se analizó con el programa Nona (Goloboff, 1999) en WinClada (Nixon, 2002); usándose la opción de búsqueda heurística, se aplicó un TBR múltiple, buscando 50000 cladogramas iniciales (mult*100) y reteniendo 20 cladogramas por réplica $(\mathrm{h} / 10)$. Se obtuvo la longitud del cladograma, así como los índices de consistencia y de retención. A partir de los cladogramas más parsimoniosos se obtuvo un cladograma de consenso estricto.

\section{Resultados}

La diversidad de las plantas vasculares acuáticas estrictas nativas en Tamaulipas es de 93 especies: 8 exclusivas de ambientes lóticos (asociadas con aguas con corrientes), 62 exclusivas de ambientes lénticos (asociadas con aguas con poco movimiento) y 23 que pueden encontrarse en ambos ambientes, distribuidas en las 7 cuencas y 15 subcuencas hidrológicas consideradas en este trabajo (Apéndice 1, Cuadro 1). La mayor riqueza se encuentra en la cuenca Río Tamesí (75 especies, $80.6 \%$ ), la cual se localiza en la porción sur del estado. Las cuencas Laguna de San Andrés y Río Soto la Marina tienen una riqueza más o menos similar, con 46 (49.5\%) y $39(41.9 \%)$ especies, respectivamente; la primera ocupa la porción sureste del estado y la segunda la parte central (Fig. 2). Por su parte, las cuencas del norte del estado tienen un menor número de especies: Río San Fernando tiene 10 (10.8\%); Laguna Madre, 27 (29\%) y Río Bravo, 23 (24.7\%). Finalmente, la Río Tula, ubicada en la porción sureste de Tamaulipas, registró la menor riqueza con sólo 3 especies (3.2\%).

Por otro lado, la distribución de la riqueza por subcuencas es muy variable, fluctuando de 3 a 68 especies 
Cuadro 2. Matriz de datos conteniendo 15 subcuencas y 98 especies de plantas vasculares acuáticas estrictas (nombres de las especies en Apéndice 1)

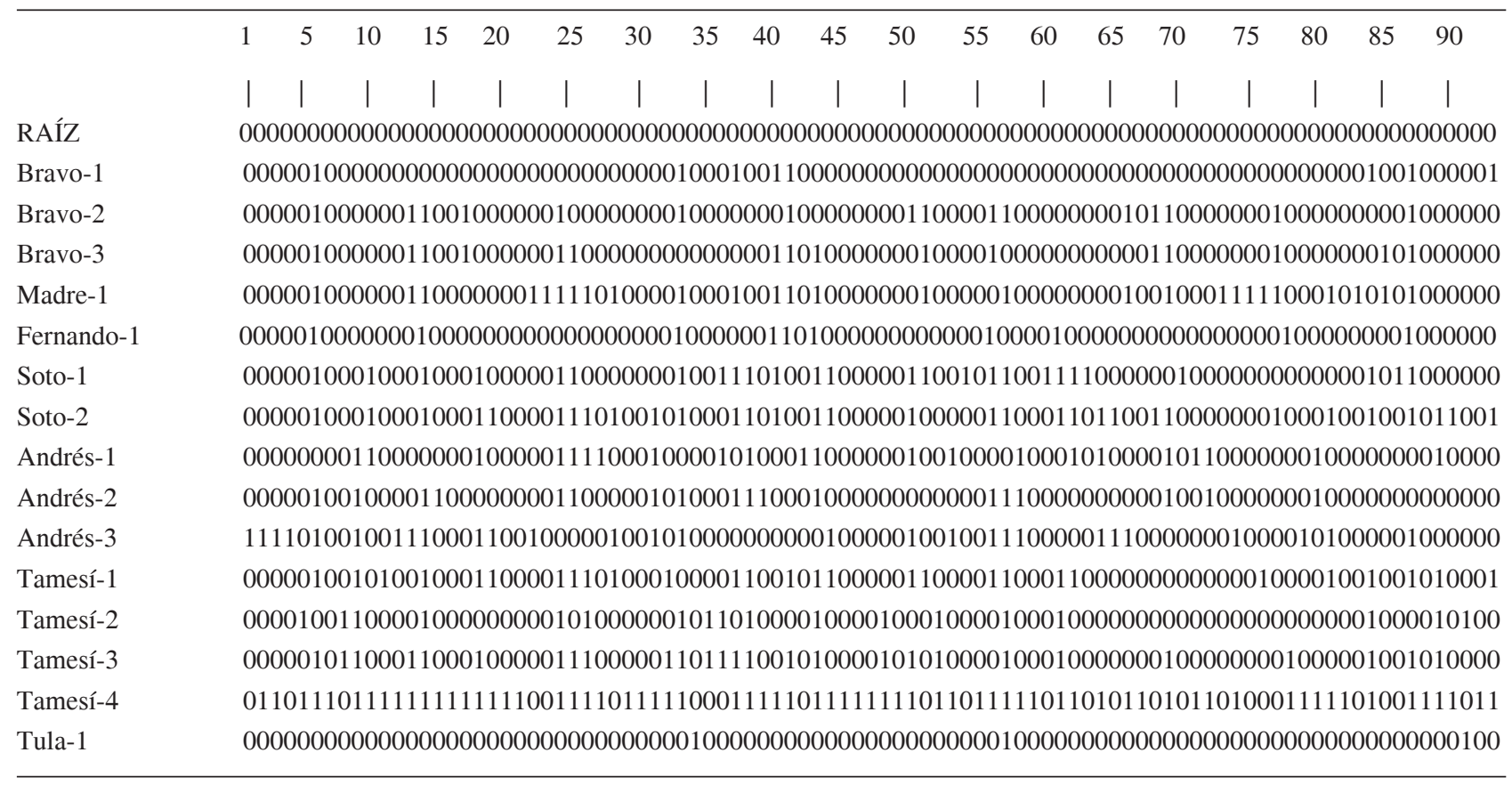

(Cuadro 1), con un promedio de 23 especies por subcuenca y una desviación estándar de 15. La subcuenca con mayor número de especies es Tamesí 4, con 68 especies (73.1\%) y la que registró menos especies es Tula 1, con 3 especies (3.2\%). El resto de las subcuencas tuvo entre 8 y 32 especies. Tamaulipas no posee especies endémicas de plantas vasculares acuáticas estrictas en su territorio, aunque se registraron 2 especies endémicas de México: Lobelia purpusii de las subcuencas Tamesí 2 y Tamesí 3, y Oserya coulteriana, restringida a la subcuenca Soto 1.

En promedio, el número de registros por subcuenca es de 92, fluctuando de 8 a 623 (Cuadro 1). Se observa una fuerte correlación entre el número de registros y el número de especies por subcuenca $\left(\mathrm{R}^{2}=0.803\right.$, $\mathrm{p}<$ 0.0001); destaca Tamesí 4, que presentó el mayor número de especies (68) y el mayor número de registros (623). Tula 1, con menos especies (3), tuvo el menor número de registros (8). La superficie de las subcuencas analizadas no es uniforme, variando de $686.7 \mathrm{~km}^{2}$ a $12146.7 \mathrm{~km}^{2}$, con un valor promedio de $5225.33 \mathrm{~km}^{2}$ (Cuadro 1). No existe correlación entre la superficie de las subcuencas y el número de especies $\left(\mathrm{R}^{2}=0.01114, \mathrm{p}=0.0172\right)$. Soto 1 , con mayor superficie (12 $\left.146.1 \mathrm{~km}^{2}\right)$, no registró la mayor riqueza de especies y Andrés 3, con menos área (686.7 $\mathrm{km}^{2}$ ), no tuvo el menor número de especies (Cuadro 1).

Se consideran como raras 29 especies (31.2\%), por presentarse en una sola subcuenca. Bacopa monnieri y Echinodorus berteroi son de distribución amplia, habitando en 12 de las 15 subcuencas. Tamesí 4 es la que registra el mayor número de especies restringidas a una subcuenca (16, 55.2\%); algunas de ellas, como Cabomba palaeformis, Gymnocoronis latifolia, Hymenachne amplexicaulis, Neptunia natans, Nymphaea amazonum y Polygonum acuminatum, son hidrófitas que alcanzan su límite de distribución septentrional en esta zona. Dentro de las especies exclusivas también se encuentra Oserya coulteriana, una hidrófita rara presente en hábitats muy restringidos, como todos los miembros de la familia Podostemaceae, la cual se registró de una pequeña porción del arroyo San Felipe, dentro de la subcuenca Soto 1.

El análisis de parsimonia de endemismos dio como resultado 6 cladogramas igualmente parsimoniosos, con una longitud de 196 pasos, un índice de consistencia de 0.47 y un índice de retención de 0.49. El cladograma de consenso estricto tuvo una longitud de 207 pasos, un índice de consistencia de 0.44 y un índice de retención de 0.44 (Fig.3). En él se reconocen varios grupos de áreas basados en la presencia de especies compartidas. El grupo I, definido por Bacopa monnieri, Lemna aequinoctialis y Typha domingensis (Cuadro 3), abarca casi todas las cuencas y subcuencas del estado, con excepción de Tula 1. El grupo II, definido por la presencia de Echinodorus berteroi, Najas guadalupensis y Paspalidium geminatum, incluye todas las subcuencas del grupo I, con excepción 


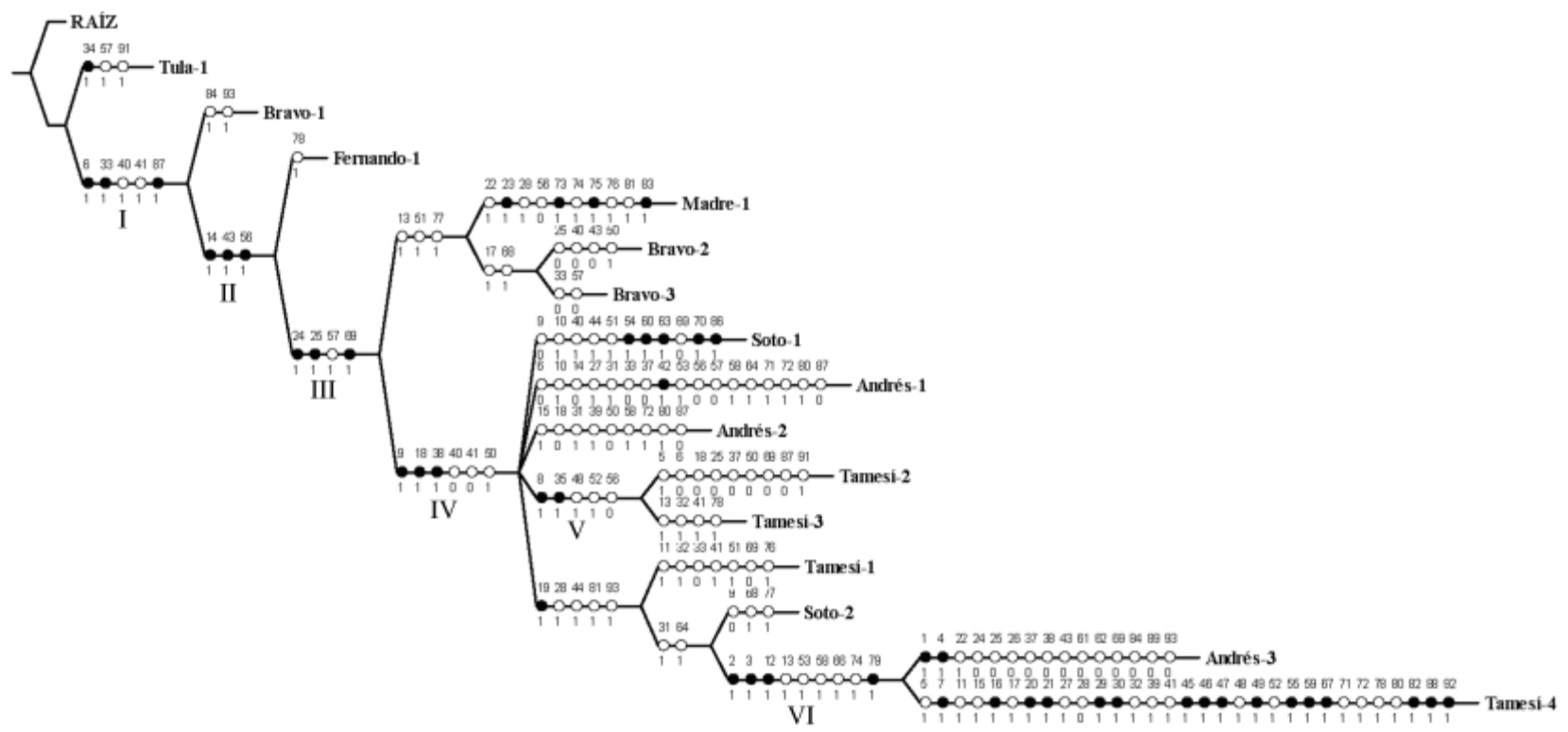

Figura 3. Cladograma de consenso estricto mostrando 6 grupos sustentados por la presencia de especies compartidas únicas. Los puntos negros indican las especies exclusivas (véase especies en el Apéndice 1 o en los Cuadros 3 y 4 ).

de Bravo 1. El grupo III, que está sustentado por Heteranthera dubia, H. limosa y Sagittaria longiloba, incluye a las subcuencas del grupo II, excepto Fernando 1 y se divide en 2 clados: el primero lo forma un grupo que, aunque no está caracterizado por especies compartidas únicas, agrupa las 3 subcuencas que conforman el área del delta del río Bravo (Madre 1, Bravo 2 y Bravo 3); mientras que el segundo clado (grupo IV) agrupa al resto de las subcuencas, está definido por Ceratophyllum demersum, Eleocharis interstincta y Ludwigia repens, y se sitúa en el centro y sureste del estado. El grupo IV está compuesto por la politomía de 5 ramas del cladograma de consenso estricto; una de ellas incluye Tamesí 2 y Tamesí 3 formando el grupo $\mathrm{V}$ y es apoyado por la presencia de 2 especies: Canna glauca y Lobelia purpusii. Otra rama sin especies compartidas, está formada por 4 subcuencas, de las cuales, Andrés 3 y Tamesí 4 forman el grupo VI que está definido por Acrostichum aureum, A. danaefolium, Crinum erubescens y Solanum tampicense.

De las 15 subcuencas analizadas, 6 presentan especies exclusivas (Cuadro 4); destaca en primer lugar Tula 1 , señalada por la presencia de Lemna gibba y que se ubica en el suroeste del estado, dentro de la amplia zona del país conocida como Altiplano Mexicano. Madre 1 incluye 4 especies propias de aguas salobres, como los pastos marinos Halophila engelmannii y Syringodium filiforme. Soto 1 se caracteriza por especies en su mayoría propias de corrientes de montaña, como las especies de Podostemaceae (Oserya coulteriana y Tristicha trifaria).
Andrés 1 registra como especie exclusiva a Myriophyllum hippuroides. Andrés 3 registra 2 especies exclusivas, propias de selvas bajas inundables que toleran aguas salobres. Tamesí 4 registra 16 especies de hidrófitas exclusivas, en su mayoría características de aguas dulces de lento movimiento (ambientes lénticos).

\section{Discusión}

La diversidad de plantas vasculares acuáticas estrictas del estado de Tamaulipas (93 especies) es menor a la registrada para el estado de Morelos (114 especies, BonillaBarbosa et al., 2000), pero mayor a la de Aguascalientes (36 especies, Siqueiros, 1989). Considerando la mayor superficie de Tamaulipas (78 $380 \mathrm{~km}^{2}$ ), comparada con la de Morelos $\left(4893 \mathrm{~km}^{2}\right)$ y Aguascalientes $\left(5618 \mathrm{~km}^{2}\right)$, además de contar una mayor cantidad de cuerpos de agua (Palacio-Prieto et al., 2000), se esperaría tener una mayor riqueza en la entidad bajo estudio. Es posible que la mayor diversidad en Morelos se deba a su posición geográfica más meridional, lo que le permite tener una mayor cantidad de especies tropicales. Dado que aún se desconoce la flora acuática estricta de otros estados del país, por el momento no se puede conocer con precisión el lugar que ocupa Tamaulipas respecto a otros estados.

La mayor riqueza de especies encontrada en ambientes lénticos (62) en comparación con los lóticos (8) concuerda con los resultados de otros trabajos, donde los cuerpos 
Cuadro 3. Especies de plantas vasculares acuáticas estrictas exclusivas a cada uno de los grupos del cladograma de consenso

\begin{tabular}{|c|c|c|}
\hline Grupo & Subcuenca* & Especies \\
\hline I & B-1, B-2, B-3, M-1, F-1, S-1, S-2, A1, A-2, A-3, T-1, T-2, T-3, T-4. & $\begin{array}{l}\text { Bacopa monnieri, Lemna aequinoctialis y Typha } \\
\text { domingensis. }\end{array}$ \\
\hline II & B-2, B-3, M-1, F-1, S-1, S-2, A1, A-2, A-3, T-1, T-2, T-3, T-4. & $\begin{array}{l}\text { Echinodorus berteroi, Najas guadalupensis y } \\
\text { Paspalidium geminatum. }\end{array}$ \\
\hline III & B-2, B-3, M-1, S-1, S-2, A1, A-2, A-3, T-1, T-2, T-3, T-4. & $\begin{array}{l}\text { Heteranthera dubia, H. limosa y Sagittaria } \\
\text { longiloba. }\end{array}$ \\
\hline IV & S-1, S-2, A1, A-2, A-3, T-1, T-2, T-3, T-4. & $\begin{array}{l}\text { Ceratophyllum demersum, Eleocharis interstincta } \\
\text { y Ludwigia repens. }\end{array}$ \\
\hline $\mathrm{V}$ & $\mathrm{T}-2, \mathrm{~T}-3$. & Canna glauca y Lobelia purpusii. \\
\hline VI & A-3, T-4 & $\begin{array}{l}\text { Acrostichum aureum, A. danaefolium, Crinum } \\
\text { erubescens y Solanum tampicense. }\end{array}$ \\
\hline
\end{tabular}

*Subcuencas: B-1 = Bravo 1, B-2 = Bravo 2, B-3 = Bravo 3, M-1 = Madre 1, F-1 = Fernando 1, S-1 = Soto 1, S-2 = Soto 2, A-1 = Andrés 1, A-2 = Andrés 2, T-1 = Tamesí 1, T-2 = Tamesí 2, Tamesí-3 = Tamesí 3, T-4 = Tamesí 4.

Cuadro 4. Subcuencas de tamaulipas, México, con especies de plantas vasculares acuáticas estrictas exclusivas

\begin{tabular}{ll}
\hline Subcuenca & Especies exclusivas \\
\hline Tula 1 & Lemna gibba. \\
Madre 1 & $\begin{array}{l}\text { Halophila engelmannii, Schoenoplectus } \\
\text { americanus, S. erectus y Syringodium } \\
\text { filiforme. }\end{array}$ \\
Soto 1 & $\begin{array}{l}\text { Oserya coulteriana, Potamogeton foliosus, } \\
\text { P. pusillus, Sagittaria platypylla y Tristicha } \\
\text { trifaria. }\end{array}$ \\
Andrés 1 & Myriophyllum hippuroides. \\
Tamesí 4 3 & $\begin{array}{l}\text { Acoelorraphe wrightii y Annona glabra. } \\
\text { Cabomba palaeformis, Echinodorus } \\
\text { grandiflorus, Gymnocoronis latifolia, } \\
\text { Habenaria repens, Hymenachne } \\
\text { amplexicaulis, Hymenocallis littoralis, Najas } \\
\text { wrightiana, Nelumbo lutea, Neptunia natans, } \\
\text { Nymphaea amazonum, Oxycarium cubense, } \\
\text { Polygonum acuminatum, Sagittaria lancifolia, } \\
\text { Stuckenia striata, Utricularia foliosa y } \\
\text { Wolfiella lingulata. }\end{array}$ \\
\hline
\end{tabular}

de agua con corriente son menos diversos que los de poco movimiento, como lagunas, estanques y charcos temporales (Martínez y García, 2001; Williams et al., 2003). Esta diferencia de riqueza también podría deberse a la mayor superficie que en Tamaulipas ocupan los cuerpos de agua lénticos en contraste con los lóticos, como sucede en el resto del país (Arriaga et al., 2000).
La distribución de especies por cuenca hidrológica indica que la mayor riqueza se concentra en la porción sureste del estado (cuenca Río Tamesí), disminuyendo paulatinamente hacia la parte norte, a excepción de Río Tula, que es la más pobre y se localiza en el Altiplano Mexicano, al suroeste del estado. El patrón de riqueza observado puede ser resultado de los niveles de precipitación, más abundantes en el sureste de Tamaulipas y menores en el norte y suroeste. La cuenca Río Tula forma parte de la región conocida como El Salado y se caracteriza por la escasa precipitación pluvial y por presentar tipos de suelo que no permiten la formación de grandes cuerpos de agua y cauces permanentes (INEGI, 2001), por lo que carece de una flora acuática diversa. Arriaga et al. (2000) señalan la cuenca del Río Tamesí como región prioritaria por su alta biodiversidad, entre otras razones por su riqueza de flora vascular acuática.

La riqueza de especies por subcuenca no está relacionada con la superficie que ocupa, más bien tiene que ver con la variedad de ambientes acuáticos presentes y el esfuerzo de colecta. La subcuenca con mayor número de especies registradas (Tamesí 4, 68 especies) incluye al amplio sistema lagunar-estuarino que forman, en su parte final, el delta de los ríos Pánuco y Tamesí en el sureste de Tamaulipas y norte de Veracruz; esta región ha sido mencionada por Rzedowski (1978) como una de las regiones del país con mayor riqueza de plantas acuáticas. Por otra parte, la recolecta de ejemplares en la subcuenca Tamesí 4 ha influido notablemente en los datos de riqueza obtenidos; trabajos previos en las lagunas del Chairel y Tancol (Mora-Olivo 1988) y en la cuenca del río Tamesí (Mora-Olivo, 2001) permitieron una exploración intensiva 
de plantas acuáticas en esta zona. Lo opuesto sucede en la subcuenca Tula 1, donde hay escasez de cuerpos de agua y se han hecho pocas recolectas.

El hecho de encontrar especies restringidas a una sola subcuenca en Tamaulipas (raras) puede deberse a distintas causas, como hábitats específicos y algunos aspectos de su distribución. Entre las especies que tienen requerimientos ecológicos especiales se encuentran Oserya coulteri y Tristicha trifaria, que sólo habitan sobre rocas en rápidos de arroyos, permaneciendo en estado vegetativo y sumergidas en la época lluviosa y expuestas al aire en la estación seca, cuando pueden producir flores y frutos, como sucede con otras especies de Podostemaceae (Novelo y Philbrick, 1997; Kita y Kato, 2004). Pocos sitios en Tamaulipas contienen ambientes adecuados para el establecimiento de poblaciones de estas especies.

La latitud define el límite septentrional o meridional de algunas especies acuáticas en Tamaulipas. Por ejemplo, Nuphar advena se distribuye desde Canadá y encuentra su límite meridional de distribución en el estado (Wiersema y Hellquist, 1997). Por otro lado, una especie neotropical que encuentra aquí su límite septentrional es Nymphaea amazonum (Mora-Olivo, 1988; RamírezGarcía y Novelo, 1989; Mora-Olivo y Villaseñor, 2007), la cual probablemente ya esté extinta, pues no se ha recolectado desde 1986 (Mahinda Martínez, com. pers.). Otro ejemplo es Tristicha trifaria, una especie propia de los trópicos de América y África (Kita y Kato, 2004), cuyo registro más al norte es en las montañas de Tamaulipas (subcuenca Soto 1). Las 2 especies endémicas de México presentes en el estado son raras; aunque una de ellas se conoce en 2 subcuencas, ambas especies tienen hábitats muy específicos: Lobelia purpusii (subcuencas Tamesí 2 y Tamesí 3), propia de arroyos de montaña y citada por McVaugh (1940) también en los estados de San Luis Potosí y Veracruz, y Oserya coulteriana se distribuye en la vertiente del Pacífico de Sonora hasta Guerrero y en Baja California Sur (Novelo y Philbrick, 1997), con una población disyunta en Tamaulipas (Mora-Olivo y Villaseñor, 2007). Estas 2 especies endémicas de México no han sido registradas en las subcuencas con mayor riqueza. Por lo general, en México la riqueza y el endemismo están correlacionados, como se observa en ciertos grupos de plantas como Asteraceae (Villaseñor et al., 1998; Balleza et al., 2005) o Cucurbitaceae (Lira et al., 2002). Es posible que las condiciones ambientales que favorecen la riqueza $\mathrm{y}$ el endemismo en plantas terrestres, sean diferentes para las plantas acuáticas. Sculthorpe (1985) menciona que quizá las especies de hidrófitas raras y endémicas tengan relación con ciertos factores físicos. De hecho, las 2 especies endémicas de México registradas en este trabajo se localizan en zonas montañosas con hábitats muy particulares. Lo anterior concuerda con la afirmación de que las montañas mexicanas son ricas en endemismos en el nivel de especie (Rzedowski, 1991a; Castillo-Campos et al., 2005).

De acuerdo con el PAE, con excepción de Tula 1, todas las subcuencas forman una gran región florística que se distingue por 3 especies compartidas cuya distribución geográfica es amplia: Bacopa monnieri, Lemna aequinoctialis y Typha domingensis. Estas especies no se registran en la subcuenca Tula 1 (que corresponde a la porción del Altiplano Mexicano), debido probablemente a que las condiciones templadas y secas allí observadas no favorecen su presencia. Algunos autores (Novelo y Lot, 2001; Lot, 2004) mencionan que Typha domingensis se distribuye preferentemente desde el nivel del mar hasta los $1000 \mathrm{~m}$ de altitud. También es conocido que las semillas de Typha domingensis pueden germinar en zonas frías, pero las plantas no maduran ni producen frutos (Smith, 2000), siendo sustituida usualmente por T. latifolia que tolera mejor las bajas temperaturas.

La exclusión de la subcuenca Bravo 1 del grupo II puede ser el resultado de la pobreza en especies observada en la parte alta de la cuenca del río Bravo, que difiere a la existente en las partes media y baja. Esta pobreza puede deberse al clima más seco en la subcuenca Bravo 1 o a las actividades humanas, como la descarga de contaminantes y la construcción de la presa Falcón, uno de los mayores embalses del país (Martínez y Novelo, 1993). De la misma manera, la exclusión de la subcuenca Fernando 1 del grupo III también puede deberse al escaso número de especies registradas.

La división del grupo III en 2 clados separa la zona del delta del río Bravo de las subcuencas más sureñas del estado. El delta del río Bravo agrupa las subcuencas Bravo 2, Bravo 3 y Madre 1, las cuales registran en conjunto 31 especies que son una mezcla de taxones de agua dulce (e.g. Echinodorus berteroi, Heteranthera dubia y Nymphaea elegans) y de agua salobre, como los pastos marinos que habitan en la Laguna Madre (Halodule wrightii, Syringodium filiforme, Halophila engelmannii y Thalassia testudinum). Para la porción del delta del río Bravo en los Estados Unidos, Richardson (1990) registra 36 especies de plantas vasculares que, de acuerdo con el criterio de Mora-Olivo y Villaseñor (2007), son acuáticas estrictas; sin embargo, sólo 21 especies se comparten entre las porciones mexicana y estadounidense. Estudios futuros podrían aumentar el número de especies para esta zona, la cual Britton y Morton (1989) señalan como un estuario con alta diversidad biológica.

El grupo IV contiene todas las subcuencas que se distribuyen desde la cuenca Río Soto la Marina, en el centro del estado, hasta la cuenca Río Tamesí que limita el 
estado de Tamaulipas con Veracruz. Esta región contiene el $93.5 \%$ de la riqueza de hidrófitas estrictas nativas registradas en Tamaulipas, incluyendo las 2 especies endémicas de México (Oserya coulteriana y Lobelia pusrpusii). De manera particular, en la cuenca Río Soto la Marina se encuentra el límite de distribución septentrional de muchas plantas acuáticas tropicales, como Tristicha trifaria, Rhizophora mangle y Laguncularia racemosa. Esta cuenca fue señalada por Gehlbach et al. (1976) como el límite noreste de las avifaunas tropicales en el Nuevo Mundo. Más recientemente, De la Maza (2005), al trabajar con peces cíclidos, menciona que "el cambio en especies de la cuenca San Fernando a la cuenca Soto La Marina está bien marcado y es abrupto"; sin embargo, algunos estudios de peces en el Golfo de México (Hulsey et al., 2004; Espinosa y Huidobro, 2005) señalan que la Faja Volcánica Transmexicana (situada aproximadamente $500 \mathrm{~km}$ al sur de la cuenca del río Soto la Marina) es el límite entre las regiones Neártica y Neotropical para estos organismos.

El grupo V incluye 2 subcuencas que ocupan porciones de la Sierra Madre Oriental conocidas como Sierra Los Nogales, Sierra Argentina, Sierra de Tamalave y Sierra de Cucharas o de Guatemala. En estas subcuencas se encuentra la Reserva de la Biosfera El Cielo, la cual es conocida ampliamente por su alta riqueza de plantas terrestres y acuáticas (Johnston et al., 1989; HernándezSandoval et al., 2005; Mora-Olivo y Novelo, 2005) y por contener cuerpos de agua en buen estado de conservación como el río Sabinas (Martínez y Novelo, 1993). El grupo VI contiene subcuencas con cuerpos de agua dulce (como el río Tamesí y las lagunas Champayán, Tancol y Vega Escondida) y salobres (como la Laguna de San Andrés, la desembocadura del río Pánuco y algunas porciones de la laguna del Chairel), por lo que se encuentran especies tanto halófitas como dulceacuícolas. Al revisar los 6 cladogramas obtenidos, puede verse que las subcuencas Andrés 1 y Andrés 2 son hermanas en 3 de los 6 cladogramas; éstas se ubican en la provincia del Golfo de México. Lo mismo sucede con Soto 1, que en 3 de los 6 cladogramas es hermana del grupo formado por Tamesí 1, Soto 2, Andrés 3 y Tamesí 4, la mayoría de ellas ubicadas en el Golfo de México, con excepción de Tamesí 1 que se ubica en la Sierra Madre Oriental.

Las plantas vasculares acuáticas estrictas en el estado de Tamaulipas presentan un patrón de distribución anidado (Fig. 4), por lo que podemos concluir que son parte de una misma unidad florística. Los 6 grupos de subcuencas mostrados en el cladograma de consenso estricto no son unidades bióticas independientes y la distribución de su riqueza se explica mejor por los gradientes altitudinales, latitudinales y climáticos mostrados por sus elementos florísticos. De esta forma, subcuencas de diferentes cuencas se agrupan con las que tienen una distribución geográficoecológica equivalente, dentro de una misma provincia.

La distribución y riqueza de la flora vascular acuática estricta de Tamaulipas permite reconocer a la entidad como una zona de particular interés biogeográfico. Esta flora presenta una combinación de especies templadas y tropicales, siendo en su mayoría de distribución tropical; sin embargo, 62 especies ( $66.7 \%$ ) extienden su distribución hasta el estado vecino de Texas en los Estados Unidos de América (Johnston, 1990). Será interesante comparar en un futuro la flora acuática de Tamaulipas con otras áreas geográficas, como el sureste de los Estados Unidos, otras partes de México, América Central y del Sur, con el objeto de profundizar en el conocimiento de la distribución de las hidrófitas de México.

\section{Agradecimientos}

El primer autor agradece al Programa de Mejoramiento del Profesorado (PROMEP) de la Universidad Autónoma de Tamaulipas y al Posgrado en Ciencias Biológicas de la Universidad Nacional Autónoma de México, por apoyar sus estudios de doctorado; y a la Comisión Nacional para el Conocimiento y Uso de la Biodiversidad, el apoyo otorgado para la realización del proyecto SO78. Los autores agradecen a Mahinda Martínez y a un revisor anónimo, por sus observaciones y sugerencias para mejorar este artículo. Enrique Ortiz amablemente elaboró las figuras.

\section{Literatura citada}

Aguilar-Aguilar, R., R. Contreras-Medina y G. SalgadoMaldonado. 2003. Parsimony analysis of endemicity (PAE) of Mexican hydrological basins based on helminth parasites of freshwater fishes. Journal of Biogeography 30:18611872 .

Aguilar-Aguilar, R., R. Contreras-Medina, A. Martínez-Aquino, G. Salgado-Maldonado y A. González-Zamora. 2005. Aplicación del análisis de parsimonia de endemismos (PAE) en los sistemas hidrológicos de México: un ejemplo con helmintos parásitos de peces dulcacuícolas. In Regionalización biogeográfica en Iberoamérica y tópicos afines, J. Llorente y J. J. Morrone (eds.). Las Prensas de Ciencias. Universidad Nacional Autónoma de México. México D.F. p. 227-239.

Arriaga, L., C. Aguilar, D. Espinosa y R. Jiménez. 1997. Regionalización ecológica y biogeográfica de México. Taller de la Comisión Nacional para el Conocimiento y Uso de la Biodiversidad (CONABIO), México, D.F.

Arriaga, L., V. Aguilar y J. Alcocer. 2000. Aguas continentales y diversidad biológica de México. Comisión Nacional para el Conocimiento y Uso de la Biodiversidad (CONABIO), 


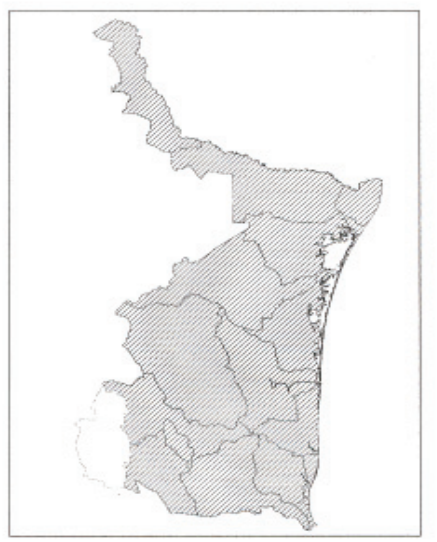

I

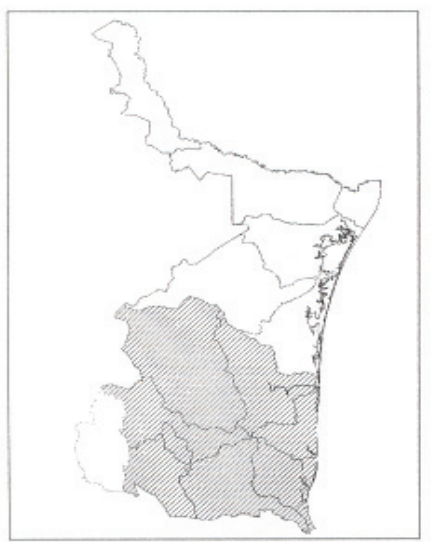

IV

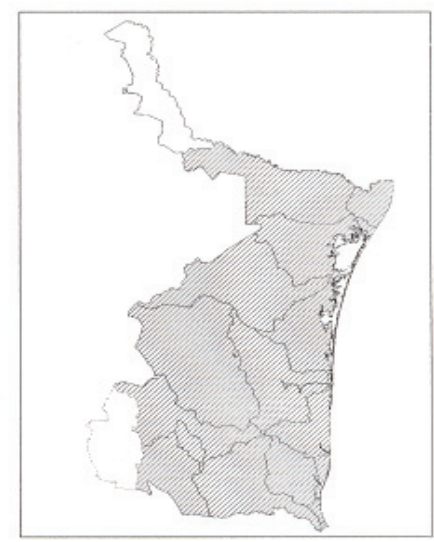

I 1

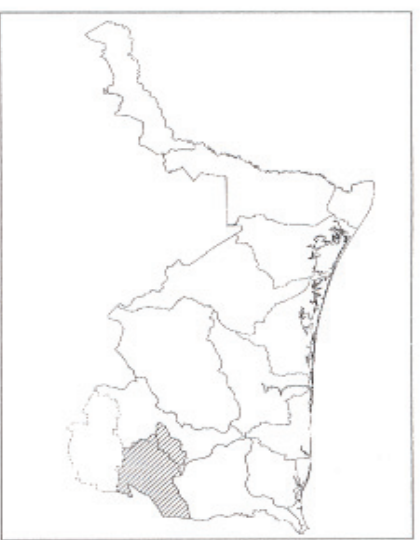

$\mathbf{V}$

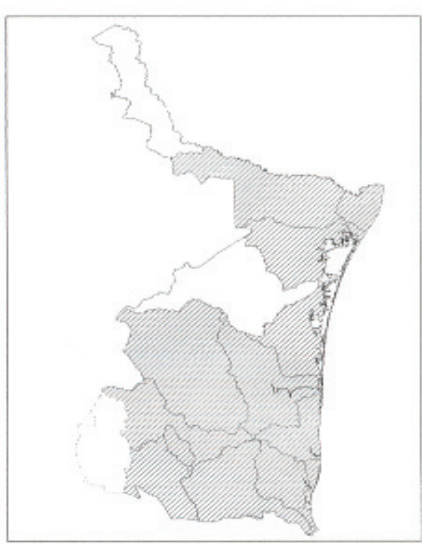

|| $\mid$

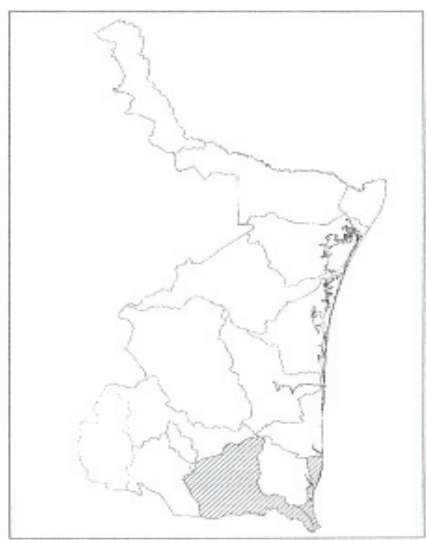

VI

Figura 4. Patrón anidado de la distribución de las plantas vasculares acuáticas estrictas en el estado de Tamaulipas con base en sus similitudes florísticas utilizando las subcuencas hidrológicas.

México, D.F. 327 p.

Balleza, J. J., J. L. Villaseñor y G. Ibarra-Manríquez. 2005. Regionalización biogeográfica de Zacatecas, México, con base en los patrones de distribución de la familia Asteraceae. Revista Mexicana de Biodiversidad 76:71-78.

Bonilla-Barbosa, J., J. A. Viana-Lases y F. Salazar-Villegas. 2005. Listados florísticos de México. XX. Flora acuática de Morelos. Instituto de Biología, Universidad Nacional Autónoma de México, México, D.F. 29 p.

Britton, J. C. y B. Morton. 1989. Shore ecology of the Gulf of Mexico. University of Texas Press. Austin. 387 p.

Casas-Andreu, G. y T. Reyna-Trujillo. 1990. Herpetofauna (anfibios y reptiles). Mapa IV.86. In Atlas Nacional de México, vol. III. Instituto de Geografía, Universidad Nacional Autónoma de México, México, D.F.

Castillo-Campos, G., M. E. Medina, P. Dávila y J. A. Zavala.
2005. Contribución al conocimiento del endemismo de la flora vascular en Veracruz, México. Acta Botanica Mexicana 73:19-57.

CNA (Comisión Nacional del Agua). 1998. Carta de regiones hidrológicas administrativas y de los estados. México, D.F.

CONABIO (Comisión Nacional para el Conocimiento y Uso de la Biodiversidad). 2007. Regionalización. URL: http://www. conabio.gob.mx/conocimiento/regionalizacion/doctos/ regionalizacion.html

Contreras-Medina, R. y H. Eliosa. 2001. Una visión panbiogeográfica preliminar de México. In Introducción a la biogeografía en Latinoamérica: Teorías, conceptos, métodos y aplicaciones, J. Llorente y J. J. Morrone (eds.). Las Prensas de Ciencias. Universidad Nacional Autónoma de México. México, D.F. p.197-211.

Contreras-Medina, R., I. Luna y J. J. Morrone. 2007a. 
Gymnosperms and cladistic biogeography of the Mexican Transition Zone. Taxon 56:905-915.

Contreras-Medina, R., I. Luna y J. J. Morrone. 2007b. Application of parsimony analysis of endemicity to Mexican gymnosperm distributions: Grid-cells, biogeographical provinces and track analysis. Biological Journal of the Linnean Society 92:405-417

Corona, A. M., R. Acosta y J. J. Morrone. 2005. Estudios biogeográficos en insectos de la Zona de Transición Mexicana. In Regionalización biogeográfica en Iberoamérica y tópicos afines, J. Llorente y J. J. Morrone (eds.). Las Prensas de Ciencias, Universidad Nacional Autónoma de México, México, D.F. p. 241-255.

Crisci, J.V., L. Katinas y P. Posadas. 2000. Introducción a la teoría y práctica de la biogeografía histórica. Sociedad Argentina de Botánica, Buenos Aires. 169 p.

Crow, G.E. 1993. Species diversity in aquatic angiosperms: latitudinal patterns. Aquatic Botany 44:229-258.

Cué-Bär, E.M., J. L. Villaseñor, J. J. Morrone y G. IbarraManríquez. 2006. Identifying priority areas for conservation in Mexican tropical deciduous forest based on tree species. Interciencia 31:712-719.

Dávila-Aranda, P., S. Arias-Montes, R. Lira-Saade, J. L. Villaseñor y A. Valiente-Banuet. 2002. Phytogeography of the columnar cacti (tribe Pachycereeae) in México: a cladistic approach. In Columnar cacti and their mutualists: evolution, ecology and conservation, T. H. Fleming y A. Valiente-Banuet (eds.). University of Arizona Press, Tucson. p. 25-41.

De la Maza, M. 2005. Adaptive radiation of the Herichthys clade in Northeastern Mexico, heroine Cichlids of Central America, Michi Tobler. URL: http://faculty-staff.ou.edu/T/ Michael.Tobler-1/cichlidae/delamaza.htm

Escalante T. y J. J. Morrone. 2003. ¿Para qué sirve el análisis de parsimonia de endemismos? In J. J. Morrone y J. Llorente. Una perspectiva latinoamericana de la biogeografía. Las Prensas de Ciencias, Universidad Nacional Autónoma de México, México, D.F. p. 167-172.

Escalante, T., G. Rodríguez, N. Cao, M.C. Ebach y J. J. Morrone. 2007. Cladistic biogeographic analysis suggests an early Caribbean diversification in Mexico. Naturwissenschaften 94:561-565.

Espadas C., R. Durán y J. Argáez. 2003. Phytogeographic analysis of taxa endemic to the Yucatán Peninsula using geographic information systems, the domain heuristic method and parsimony analysis of endemicity. Diversity and Distributions 9:313-330.

Espinosa, D., J. Llorente y J. J. Morrone. 2006. Historical biogeographic patterns of the species of Bursera (Burseraceae) and their taxonomical implications. Journal of Biogeography 33:1945-1958

Espinosa, H. y L. Huidobro. 2005. Ictiogeografía de los peces dulceacuícolas de la vertiente del Golfo de México. In Regionalización biogeográfica en Iberoamérica y tópicos afines. J. Llorente y J. J. Morrone (eds.). Las Prensas de Ciencias. Universidad Nacional Autónoma de México. México, D.F. p. 295-318.

Ferrusquía-Villafranca, I. 1990. Regionalización biogeográfica.
Mapa IV.8.10. In Atlas nacional de México, vol. III. Instituto de Geografía, Universidad Nacional Autónoma de México. México, D.F.

García-Trejo, E. A. y A. G. Navarro-Sigüenza. 2004. Patrones biogeográficos de la riqueza de especies y el endemismo de la avifauna del oeste de México. Acta Zoológica Mexicana (n.s.) 20:167-185.

Gehlbach, F. R., D. O. Dillon, H. L. Harrell, S. E. Kennedy y K. R. Wilson. 1976. Avifauna of the Rio Corona, Tamaulipas, Mexico: Northeastern limit of the tropics. The Auk 93:5365.

Glasby, C. J. y B. Álvarez. 1999. Distribution patterns and biogeographical analysis of Austral Polycheta (Annelida). Journal of Biogeography 26:507-533.

Goloboff, P. 1999. NONA, version 2.0. Publicado por el autor. San Miguel de Tucumán.

González, M. 1989. El género Potamogeton (Potamogetonaceae) en México. Acta Botanica Mexicana 6:1-43.

Haynes, R. R. y L. Holm-Nielsen. 1994. Alismataceae. Flora Neotropica Monograph 64. New York Botanical Garden. New York. 112 p.

Haynes, R. R. y L. Holm-Nielsen. 2003. Potamogetonaceae. Flora Neotropica Monograph 85. New York Botanical Garden, New York. 52 p.

Hernández-Sandoval, L. G., J. Treviño C., A. Mora-Olivo y M. Martínez. 2005. Diversidad florística y endemismos. In Historia natural de la Reserva de la Biosfera El Cielo, Tamaulipas, México, G. Sánchez-Ramos, P. Reyes-Castillo y R Dirzo (eds.). Universidad Autónoma de Tamaulipas, Ciudad Victoria. p. 244-259.

Huidobro, L., J. J. Morrone, J. L. Villalobos y F. Álvarez. 2006. Distributional patterns of freshwater taxa (fishes, crustaceans and plants) from the Mexican transition zone. Journal of Biogeography 33:731-741.

Hulsey, C. D., F. J. García de Léon, Y. Sánchez J., D. A. Hendrickson y T. J. Near. 2004. Temporal diversification of Mesoamerican cichlid fishes across a major biogeographic boundary. Molecular Phylogenetics and Evolution 31:754764.

INEGI. 2001. Síntesis geográfica del Estado de Tamaulipas. Secretaría de Programación y Presupuesto, México, D.F. $168 \mathrm{p}$.

Johnston, M. C., K. Nixon, G. L. Nesom y M. Martínez. 1989. Listado de plantas vasculares conocidas de la sierra de Guatemala, Gómez Farías, Tamaulipas, México. Biotam 1:21-33

Johnston, M. C. 1990. The vascular plants of Texas. A list, updating the Manual of the vascular plants of Texas, segunda edición. Publicado por el autor. Austin, Texas. 107 p.

Kita, Y. y M. Kato. 2004. Phylogenetic relationships between disjuntly occurring groups of Tristicha trifaria (Podostemaceae). Journal of Biogeography 31:1605-1612.

León-Paniagua, L., E. García-Trejo, J. Arroyo-Cabrales y S. Castañeda-Rico. 2004. Patrones biogeográficos de la mastofauna. In Biodiversidad de la Sierra Madre Oriental, I. Luna, J. J. Morrrone y D. Espinosa (eds.). Las Prensas de Ciencias. Universidad Nacional Autónoma de México, México, D.F. p. 469-479. 
Les, D. H., D. J. Crawford, R. T. Kimball, M. L. Moody y E. Landolt. 2003. Biogeography of discontinuously distributed hydrophytes: A molecular appraisal of intercontinental disjunctions. International Journal of Plant Sciences 164:917932.

Lira, R., J. L. Villaseñor y E. Ortiz. 2002. A proposal for the conservation of the family Cucurbitaceae in Mexico. Biodiversity and Conservation 11:1699-1720.

Lot, A., A. Novelo y P. Ramírez-García. 1986. Listados florísticos de México. V. Angiospermas acuáticas mexicanas 1. Instituto de Biología, Universidad Nacional Autónoma de México,. México, D.F. 60 p.

Lot, A. y A. Novelo. 1992. Afinidades florísticas de las monocotiledóneas acuáticas mesoamericanas. In Biogeography of Mesoamerica, S. P. Darwin y A. L. Welden (eds.). Tulane Studies in Zoology and Botany, Supplementary Publication 1. p. 147-153.

Lot, A., A. Novelo, M. Olvera y P. Ramírez-García. 1999. Catálogo de angiospermas acuáticas de México. Hidrófitas estrictas emergentes, sumergidas y flotantes. Cuadernos 33. Instituto de Biología, Universidad Nacional Autónoma de México, México, D.F. 161 p.

Lot, A. 2004. Fanerógamas acuáticas. In Biodiversidad de Oaxaca, A. J. García-Mendoza, M. J. Ordóñez y M. BrionesSalas (eds.). Instituto de Biología, Universidad Nacional Autónoma de México-Fondo para la Conservación de la Naturaleza-World Wildlife Found, México, D.F. p. 237248.

Luis, A., J. Llorente e I. Vargas. 2005. Una megabase de datos de mariposas y la regionalización biogeográfica de México. In Regionalización biogeográfica en Iberoamérica y tópicos afines, J. Llorente y J. J. Morrone (eds.). Las Prensas de Ciencias. Universidad Nacional Autónoma de México, México, D.F. p. 269-294.

Luna, I., O. Alcántara, D. Espinosa y J. J. Morrone. 1999. Historical relationships of the Mexican cloud forest: a preliminary vicariance model applying parsimony analysis of endemicity to vascular plant taxa. Journal of Biogeography 26:1299-1305.

Luna, I., O. Alcántara, D. Espinosa y J. J. Morrone. 2000. Track analysis and conservation priorities in the cloud forest of Hidalgo, Mexico. Diversity and Distributions 6:137-143.

Martínez, M. y A. Novelo. 1993. La vegetación acuática del estado de Tamaulipas, México. Anales del Instituto de Biología, Universidad Nacional Autónoma de México, Serie Botánica 64:59-86.

Martínez, M. y A. García M. 2001. Flora y vegetación acuáticas de localidades selectas del estado de Querétaro. Acta Botanica Mexicana 54:1-23.

Méndez-Larios, I., J. L. Villaseñor, R. Lira, J. J. Morrone, P. Dávila y E. Ortiz. 2005. Toward the identification of a core zone in the Tehuacán-Cuicatlán Biosphere Reserve, Mexico, based on parsimony analysis of endemicity of flowering plant species. Interciencia 30:267-274.

McGlone, M.S., R.P. Duncan y P.B. Heenan. 2001. Endemism, species selection and the origin and distribution of the vascular plant flora of New Zealand. Journal of Biogeography 28: 199-216.
McVaugh, R. 1940. A key to the North American species of Lobelia (Sect. Hemipogon). American Midland Naturalist 24:681-702.

Miranda, F. y E. Hernández-X. 1963. Los tipos de vegetación de México y su clasificación. Boletín de la Sociedad Botánica de México 28:29-179.

Mora-Olivo, A. 1988. Plantas acuáticas y semiacuáticas de las lagunas del Chairel y Tancol, Tampico, Tamaulipas, México. Tesis, Escuela de Ciencias Biológicas, Universidad del Noreste, Tampico, Tamaulipas. 201 p.

Mora-Olivo, A. 2001. Informe técnico final del Proyecto S078 flora vascular acuática de la cuenca del río Tamesí. Universidad Autónoma de Tamaulipas, Ciudad Victoria. 25 p.

Mora-Olivo, A. y A. Novelo. 2005. La vegetación acuática y semiacuática. In Historia natural de la Reserva de la Biosfera El Cielo, Tamaulipas, México, G. Sánchez-Ramos, P. Reyes-Castillo y R Dirzo (eds.). Universidad Autónoma de Tamaulipas, Ciudad Victoria. p. 106-115.

Mora-Olivo, A. y J. L. Villaseñor. 2007. Diversidad y distribución de la flora vascular acuática de Tamaulipas, México. Journal of the Botanical Research Institute of Texas 1:511-527.

Morrone, J. J. 1994. On the identification of areas of endemism. Systematic Biology 43:438-441.

Morrone, J. J. y J. V. Crisci. 1995. Historical biogeography: Introduction to methods. Annual Review of Ecology and Systematics 26:373-401.

Morrone, J. J., D. Espinosa O. y J. Llorente. 2002. Mexican biogeographic provinces: Preliminary scheme, general characterizations, and synonymies. Acta Zoologica Mexicana (n.s.) 85:83-108.

Murguía, M. y F. Rojas. 2001. Biogeografía cuantitativa. In Introducción a la biogeografía en Latinoamérica: teorías, conceptos, métodos y aplicaciones, J. Llorente y J. J. Morrone (eds.). Las Prensas de Ciencias, Universidad Nacional Autónoma de México, México, D.F. p. 39-47.

Nixon, K.C. 2002. WinClada, ver. 1.00.08. Ithaca. www. cladistics.com/about_winc.htm

Novelo, A. y A. Lot. 2001. Typhaceae. In Flora fanerogámica del Valle de México, G. Calderón de Rzedowski y J. Rzedowski (eds.). Instituto de Ecología, Pátzcuaro, Michoacán. p. 978980.

Novelo, R., A. y C. T. Philbrick. 1997. Taxonomy of Mexican Podostemaceae. Aquatic Botany 57:37-50.

Olvera, M. 1996. El género Utricularia (Lentibulariaceae) en México. Anales del Instituto de Biología, Universidad Nacional Autónoma de México, Serie Botánica 67:347-384.

Palacio-Prieto, J. L., G. Bocco, A. Velásquez, J. F. Mas, F.Takaki-Takaki, L. Luna-González, G. Gómez-Rodríguez, J. López-García, M. Palma M., I. Trejo V., A. Peralta H., J. Prado-Molina, A. Rodríguez-Aguilar, R. Mayorga-Saucedo y F. González M. 2000. La condición actual de los recursos forestales en México: resultados del Inventario Forestal Nacional 2000. Investigaciones Geográficas, Boletín del Instituto de Geografía, Universidad Nacional Autónoma de México 43:183-203.

Pérez-García, B., R. Riba y D. M. Johnson. 1999. Pteridofitas. Familia Marsileaceae. Flora de México 6:1-17.

Ramírez-García, P. y A. Novelo R. 1989. Nymphaea amazonum 
(Nymphaeaceae) en México: Clave de las especies del subgénero Hydrocallis en el país. Anales del Instituto de Biología Universidad Nacional Autónoma de México, Serie Botánica 58:87-92.

Ramírez-Pulido, J. y A. Castro-Campillo. 1990. Regionalización mastofaunística (mamíferos). Mapa IV.8.8.A. In Atlas nacional de México, vol. III. Instituto de Geografía, Universidad Nacional Autónoma de México, México, D.F.

Richardson, A. 1990. Plants of the Rio Grande delta. University of Texas Press, Austin. 94 p.

Rosen, B. R. 1988. From fossils to earth history: applied historical biogeography. In. Analytical biogeography: an integrated approach to the study of animal and plant distributions, A. A. Myers y P. S. Giller (eds.). Chapman and Hall, Londres. p. 437-481.

Rzedowski, J. 1978. La vegetación de México. Limusa, México, D.F. 432 p.

Rzedowski, J. 1991a. Diversidad y orígenes de la flora fanerogámica de México. Acta Botanica Mexicana 14:3-21.

Rzedowski, J. 1991b. El endemismo en la flora fanerogámica mexicana: una apreciación analítica preliminar. Acta Botanica Mexicana 15:47-64.

Santamaría, L. 2002. Why are most aquatic plants widely distributed? Dispersal, clonal growth and small-scale heterogeneity in a stressful environment. Acta Oecologica 23:137-154.

Santamaría, L. y M. Klaassen. 2002. Waterbird-mediated dispersal of aquatic organisms: An introduction. Acta Oecologica 23:115-119.

SAS. 2002. JMP Version 5. SAS Institute Inc. http://www.jmp.com Sawada, M., A. E. Viau y K. Gajewski. 2003. The biogeography of aquatic macrophytes in North America since the last glacial maximum. Journal of Biogeography 30:999-1071.

Sculthorpe, C.D. 1985. The biology of aquatic vascular plants. Edward Arnold, Londres. 610 p.

Siqueiros, M. E. 1989. Contribución a la flora acuática y subacuática de Aguascalientes. Universidad Autónoma de Aguascalientes, Aguascalientes. 75 p.

Smith, S.G. 2000. Typhaceae. In Flora of North America North of Mexico, vol. 22, Flora of North America Editorial Committee (eds.) Oxford University Press, New York. p. 278-285.

SPP (Secretaría de Programación y Presupuesto). 1982. Carta estatal hidrológica superficial. Escala 1:250,000. México, D.F.

Stuckey, R. L. 1993. Phytogeographical outline of aquatic and wetland angiosperms in continental eastern North America. Aquatic Botany 44:259-301.

Tucker, G. C. 1994. Revision of the Mexican species of Cyperus (Cyperaceae). Systematic Botany Monographs 43:1-43.

Villaseñor, J. L., G. Ibarra-Manríquez y D. Ocaña. 1998. Strategies for the conservation of Asteraceae in Mexico. Conservation Biology 12:1066-1075.

Williams, P., M. Whitfield, J. Biggs, A. Bray, G. Fox, P. Nicolet y D. Sear. 2003. Comparative biodiversity of rivers, streams, ditches and ponds in an agricultural landscape in southern England. Biological Conservation 115:329-341.

Wiersema, J.H. y C.B. Hellquist. 1997. Nymphaeaceae. In Flora of North America North Mexico, vol. 3, Flora of North America Editorial Committee (eds.) Oxford University Press, New York. p. 66-77.

Zunino, M. 2005. Corotipos y biogeografía sistemática en el Euromediterráneo. In Regionalización biogeográfica en Iberoamérica y tópicos afines, J. Llorente y J. J. Morrone (eds.). Las Prensas de Ciencias. Universidad Nacional Autónoma de México. México, D.F. p. 181-187.

Apéndice 1. Especies de las plantas vasculares acuáticas estrictas de Tamaulipas, indicando el tipo de ambiente que ocupan en el estado de Tamaulipas. $\mathrm{L}=$ Ambiente léntico, $\mathrm{O}=$ Ambiente lótico, y $\mathrm{L} / \mathrm{O}=$ Ambientes léntico y lótico

1. Acoelorraphe wrightii (Griseb. et H. Wendl.) H. Wendl. ex

Becc. (Arecaceae) L

2. Acrostichum aureum L. (Polypodiaceae) L

3. Acrostichum danaeifolium Langsd. et Fisch. (Polypodiaceae) L/O

4. Annona glabra L. (Annonaceae) L/O

5. Azolla microphylla Kaulf. (Azollaceae) L

6. Bacopa monnieri (L.) Wettst. (Scrophulariaceae) L/O

7. Cabomba paleaformis Fassett. (Cabombaceae) L

8. Canna glauca L. (Cannaceae) L

9. Ceratophyllum demersum L. (Ceratophyllaceae) L/O

10. Ceratopteris thalictroides (L.) Brongn. (Parkeriaceae) L

11. Cladium jamaicense Crantz. (Cyperaceae) L/O

12. Crinum erubescens Sol. (Amaryllidaceae) L/O

13. Cyperus articulatus L. (Cyperaceae) L
14. Echinodorus berteroi (Spreng.) Fassett. (Alismataceae) L

15. Echinodorus cordifolius (L.) Griseb. (Alismataceae) L

16. Echinodorus grandiflorus (Cham. et Schltdl.) Micheli.

(Alismataceae) L

17. Eleocharis acicularis (L.) Roem. et Schult. (Cyperaceae) L

18. Eleocharis interstincta (Vahl) Roem. et Schult. (Cyperaceae) L

19. Eleocharis mutata (L.) Roem. et Schult. (Cyperaceae) L

20. Gymnocoronis latifolia Hook. et Arn. (Asteraceae) L

21. Habenaria repens Nutt. (Orchidaceae) L

22. Halodule wrightii Asch. (Cymodoceaceae) L

23. Halophila engelmannii Asch. (Hydrocharitaceae) L

24. Heteranthera dubia (Jacq.) MacMill. (Pontederiaceae) L/O

25. Heteranthera limosa (Sw.) Willd. (Pontederiaceae) L

26. Heteranthera mexicana S. Watson.(Pontederiaceae) L 
27. Heteranthera rotundifolia (Kunth) Griseb. (Pontederiaceae) L

28. Hydrolea spinosa L. (Hydrophyllaceae) L

29. Hymenachne amplexicaulis (Rudge) Nees. (Poaceae) L

30. Hymenocallis littoralis (Jacq.) Salisb. (Amaryllidaceae) L

31. Laguncularia racemosa (L.) C.F. Gaertn. (Combretaceae) L/O

32. Leersia hexandra Sw. (Poaceae) L

33. Lemna aequinoctialis Welw. (Lemnaceae) L

34. Lemna gibba L. (Lemnaceae) L

35. Lobelia purpusii Brandegee (Campanulaceae) $\mathrm{O}$

36. Ludwigia palustris (L.) Elliott (Onagraceae) $\mathrm{O}$

37. Ludwigia peploides (Kunth) P.H. Raven (Onagraceae) L/O

38. Ludwigia repens J.R. Forst. (Onagraceae) O

39. Marsilea ancylopoda A. Braun (Marsileaceae) L

40. Marsilea macropoda A. Braun (Marsileaceae) L

41. Marsilea vestita Hook. et Grev. (Marsileaceae) L

42. Myriophyllum hippuroides Nutt. ex Torr. et A.Gray

(Haloragaceae) $\mathrm{O}$

43. Najas guadalupensis (Spreng.) Magnus (Najadaceae) L/O

44. Najas marina L. (Najadaceae) L/O

45. Najas wrightiana A. Braun (Najadaceae) L

46. Nelumbo lutea (Willd.) Pers. (Nelumbonaceae) L

47. Neptunia natans (L. f.) Druce (Mimosaceae) L

48. Nuphar advena (Aiton) W.T. Aiton (Nymphaeaceae) O

49. Nymphaea amazonum Mart. et Zucc. (Nymphaeaceae) L

50. Nymphaea ampla (Salisb.) DC. (Nymphaeaceae) L

51. Nymphaea elegans Hook. (Nymphaeaceae) L

52. Nymphaea jamesoniana Planch. (Nymphaeaceae) L

53. Nymphoides indica (L.) Kuntze. (Menyanthaceae) L

54. Oserya coulteriana Tul. (Podostemaceae) $\mathrm{O}$

55. Oxycarium cubense (Poepp. et Kunth) Lye. (Cyperaceae) L

56. Paspalidium geminatum (Forssk.) Stapf. (Poaceae) L

57. Paspalum distichum L. (Poaceae) L/O

58. Pistia stratiotes L. (Araceae) L

59. Polygonum acuminatum Kunth. (Polygonaceae) L

60. Potamogeton foliosus Raf. (Potamogetonaceae) L/O

61. Potamogeton illinoensis Morong. (Potamogetonaceae) L/O
62. Potamogeton nodosus Poiret (Potamogetonaceae) L/O

63. Potamogeton pusillus L. (Potamogetonaceae) L/O

64. Rhizophora mangle L. (Rhizophoraceae) L/O

65. Rotala ramosior (L.) Koehne. (Lythraceae) L

66. Ruppia maritima L. (Ruppiaceae) L

67. Sagittaria lancifolia L. (Alismataceae) L

68. Sagittaria latifolia Willd. (Alismataceae) L

69. Sagittaria longiloba Engelm. ex J.G. Sm. (Alismataceae) L

70. Sagittaria platyphylla (Engelm.) J.G. Sm. (Alismataceae) L

71. Salvinia auriculata Aubl. (Salviniaceae) L

72. Salvinia minima Baker (Salviniaceae) L

73. Schoenoplectus americanus (Pers.) Volkart ex Schinz et R.

Keller (Cyperaceae) L/O

74. Schoenoplectus californicus (C.A. Mey.) Soják

(Cyperaceae) L/O

75. Schoenoplectus erectus (Poir.) Palla ex J. Raynal

(Cyperaceae) L

76. Schoenoplectus pungens (Vahl) Palla. (Cyperaceae) L

77. Schoenoplectus saximontanus (Fernald) J. Raynal

(Cyperaceae) L

78. Schoenoplectus tabernaemontani (C.C. Gmel.) Palla.

(Cyperaceae) L/O

79. Solanum tampicense Dunal (Solanaceae) L

80. Spirodela polyrhiza (L.) Schleid. (Lemnaceae) L

81. Stuckenia pectinata (L.) Börner (Potamogetonaceae) L

82. Stuckenia striata (Ruiz et Pav.) Holub. (Potamogetonaceae) L

83. Syringodium filiforme Kütz. (Cymodoceaceae) L

84. Taxodium mucronatum Ten. (Taxodiaceae) $\mathrm{O}$

85. Thalassia testudinum Banks ex König (Hydrocharitaceae) L

86. Tristicha trifaria (Bory ex Willd.) Spreng. (Podostemaceae) O

87. Typha domingensis Pers. (Typhaceae) L/O

88. Utricularia foliosa L. (Lentibulariaceae) L

89. Utricularia gibba L. (Lentibulariaceae) L

90. Vallisneria americana Michx. (Hydrocharitaceae) L/O

91. Wolffia brasiliensis Wedd. (Lemnaceae) L

92. Wolffiella lingulata (Hegelm.) Hegelm. (Lemnaceae) L

93. Zannichellia palustris L. (Zannichelliaceae) L/O 